\title{
Time course from first symptom to treatment in patients with non-small cell lung cancer referred for radiotherapy: a report by the CHART Steering Committee
}

\author{
S Dische, D Gibson, M Parmar, M I Saunders on behalf of the CHART Steering \\ Committee
}

Members of the CHART Steering Committee: A Barrett (chairman) $S$ Dische (trial coordinator), M I Saunders (trial co-ordinator), B Cottier, M Davies, $P$ Dawes, $M$ F Drummond, C Gaffney, $T$ Hince, $T$ Herrman, $\mathrm{J}$ M Henk, F Macbeth, W Maton-Howarth, D A L Morgan, H Newman, J R Roberts, R I Rothwell, V H Svoboda, R P

Symonds, J S Tobias, $M$ $\mathrm{H}$ Whipp, H Yosef, B Littbrand, C K Bomford, D Coyle, A Galpine, A

G Robertson, $M$ H

Robinson, J Shaw, C

Williams, E Wilson, R G

Aitken. Data

Management,

Computing and

Statistics: M Parmar

(statistician), D Gibson,

A Harvey (data

managers)

Correspondence to:

Dr M Parmar, MRC Cancer

Trials Office, 5 Shaftesbury

Road, Cambridge CB2 2BW,

UK

Received 3 July 1995

Returned to author

30 August 1995

Revised version received

13 May 1996

Accepted for publication

18 June 1996

\begin{abstract}
Background - Only a small proportion of patients with non-small cell lung cancer (NSCLC) attending radiotherapy centres were suitable for inclusion in a randomised trial which compared continuous hyperfractionated accelerated radiotherapy (CHART) with conventional radical radiotherapy. As this was thought to be partly due to delays in the referral of patients to clinical oncologists, a prospective study was performed to determine the interval between first report of symptoms and first radiotherapy treatment in patients with NSCLC.

Methods - The time course from first symptom to treatment was determined in all patients with NSCLC attending 10 cancer centres for radiotherapy to a primary tumour in a three month period.

Results - Only $5 \%$ of 484 patients were suitable for the trial of radical radiotherapy. The principal causes for exclusion were poor general condition (37\%), too large a tumour $(27 \%)$, and extrathoracic metastases (19\%). The median time from first symptom to diagnosis was 13 weeks, from first symptom to first treatment 19 weeks, and from diagnosis to first treatment five weeks. In a quarter of the patients these intervals were greater than 25,33 weeks, and nine weeks, respectively. Conclusion - The reason for these long intervals needs investigation since earlier diagnosis and more immediate referral for consideration of treatment might increase the number of patients with NSCLC suitable for radical radiotherapy.

(Thorax 1996;51:1262-1265)
\end{abstract}

Keywords: non-small cell lung cancer, radiotherapy, CHART, time to treatment.

Lung cancer is the most commonly occurring malignancy in the European Community with 160000 new cases every year. ${ }^{1}$ Patients undergoing complete surgical resection have a 30 $40 \%$ chance of gaining long term survival, but they must be fit for surgery and the tumour must be localised to permit complete removal. It is estimated that only $10 \%$ of patients with lung cancer undergo pulmonary resection in the United Kingdom. ${ }^{2}$ Approximately $80 \%$ of all lung cancers are of the non-small cell histological type (NSCLC). ${ }^{3}$ Unfortunately, long term survival in this disease is generally poor with only $8 \%$ of all cases alive at five years. ${ }^{4}$

There are a few patients who present with small early tumours who do not have surgery, either because of the site of the tumour or because the patient is medically unsuitable, or because the patient refuses surgery. In such cases high dose (radical) radiotherapy may achieve results comparable to those in resected cases. ${ }^{5}$ However, the number of patients currently treated with high dose radiotherapy is small and makes no impact upon the general problem of the management of lung cancer.

A much larger number of patients with locally advanced inoperable tumours show no evidence of distant spread at the time of investigation. When these patients are referred to centres in the UK the general policy is to employ low dose radiotherapy with palliative intent. In North America and in continental Europe high doses are commonly given but the value in terms of primary tumour control or cure is arguable.

In a pilot study of continuous hyperfractionated accelerated radiotherapy (CHART) performed at Mount Vernon Hospital 78 patients with NSCLC presenting with locally advanced disease (62 in stage T3 and 44 in N2) showed one year and two year survival rates of $65 \%$ and $32 \%$. Historical data for patients treated by previously employed schedules of radiotherapy showed one and two year survival rates of $40 \%$ and $15 \%$, figures comparable to those recorded in other series. ${ }^{6}$ In 1990 the CHART Steering Committee, established jointly by the Cancer Research Campaign, Medical Research Council, and Department of Health, organised randomised controlled clinical trials of the CHART regime in head and neck cancer and in non-small cell lung cancer. In these trials a comparison was made with patients treated by conventional schemes of radical radiotherapy considered to follow the best international practice. ${ }^{7}$

The essential requirements for inclusion in the lung cancer trial were a WHO performance status 0 or 1 and a locally advanced NSCLC unsuitable for surgery with no evidence of metastases outside the thorax. The area to be treated within the chest, when viewed anteriorly, was not to exceed $240 \mathrm{~cm}^{2}$ so that a radical course of radiotherapy could be given without risk of 
serious impairment of lung function. Entry to the study began in February 1990 and by March 1995 a total of 563 patients with NSCLC had been entered by the 13 centres participating in the study.

The rate of accrual of patients with lung cancer was slower than those with head and neck cancer where, in the same period of time, 918 patients were entered. It was reported from all the centres that most patients with NSCLC were not included because they did not satisfy the criteria for entry. A survey in Glasgow suggested that fewer than $5 \%$ of cases presenting to the cancer centre could be included; most commonly the tumours were too advanced within the chest, or the patient's general condition was too poor (Macbeth, personal communication).

Because of the view held generally in the UK that patients with locally advanced disease should only be given palliative radiotherapy, the CHART Steering Committee felt that there might be delays in referrals so that some patients who were suitable for radical radiotherapy at the time of presentation to their general practitioner no longer fulfilled the requirements for inclusion when finally seen by a clinical oncologist. Further, it was considered important that the patients included in the randomised trial be placed in the context of all those presenting to cancer centres with the same clinical condition. It was decided therefore to perform a survey of all patients with lung cancer who received any radiotherapy to the primary tumour at the cancer centres contributing to the CHART trials.

\section{Methods}

All 13 centres contributing patients to the CHART trial (10 UK, three non-UK) participated in the survey. This began on 1 November 1993 and covered a three month period ending on 31 January 1994 except at one centre which surveyed the period from 1 October to 31 December 1993.

All patients with NSCLC referred for and treated by radiotherapy to the primary tumour were included in the analysis but we excluded those who came for radiotherapy for distant metastases, those who came for chemotherapy, and those in whom only supportive care was advised at the cancer centre.

A trial co-ordinator (nurse or therapy radiographer) collected the data in each centre. The dates of onset of symptoms, first attendance at a general practitioner, hospital, oncologist, and of the start of treatment were determined The hospital notes and referral letters were available to the local trial co-ordinators for the data to be extracted and, where necessary, they consulted the clinical oncologist. In addition, the dose and fractionation of the radiotherapy given was recorded and, in those excluded from the CHART trial, the principal reason for that decision. It was felt realistic only to request the month and year of onset of the first symptom whereas with the other data the day was recorded.
As the data were gathered at the time when the patients were being prepared for treatment it was possible to check dates with them. The date of diagnosis was, in most cases, the date when histological or cytological proof of diagnosis was available. In some of the more advanced cases, however, histological or cytological proof was not obtained and the diagnosis rested on the clinical findings. In such a case the date given was the one where the diagnosis was considered so certain that management decisions could be made.

\section{Results}

In one large centre less than $10 \%$ of the expected number of forms were completed and the data from this centre were therefore excluded from the analysis.

A total of 524 patients receiving radiotherapy to the primary tumour during the three month period of the survey were recorded in the remaining 12 centres. Of these, 40 had first undergone tumour resection and these have been excluded from further analysis leaving 484 cases (table 1). A further 34 patients were included who had undergone thoracotomy but in whom resection was not possible. The numbers contributed by each centre varied widely reflecting both the population served and the number of patients contributed annually to the randomised trial.

Patient details are shown in table 1. Men outnumbered women by $3: 1$ and $60 \%$ of the patients were under the age of 70 . The radiotherapy was given in three or fewer fractions

Table 1 Patient details

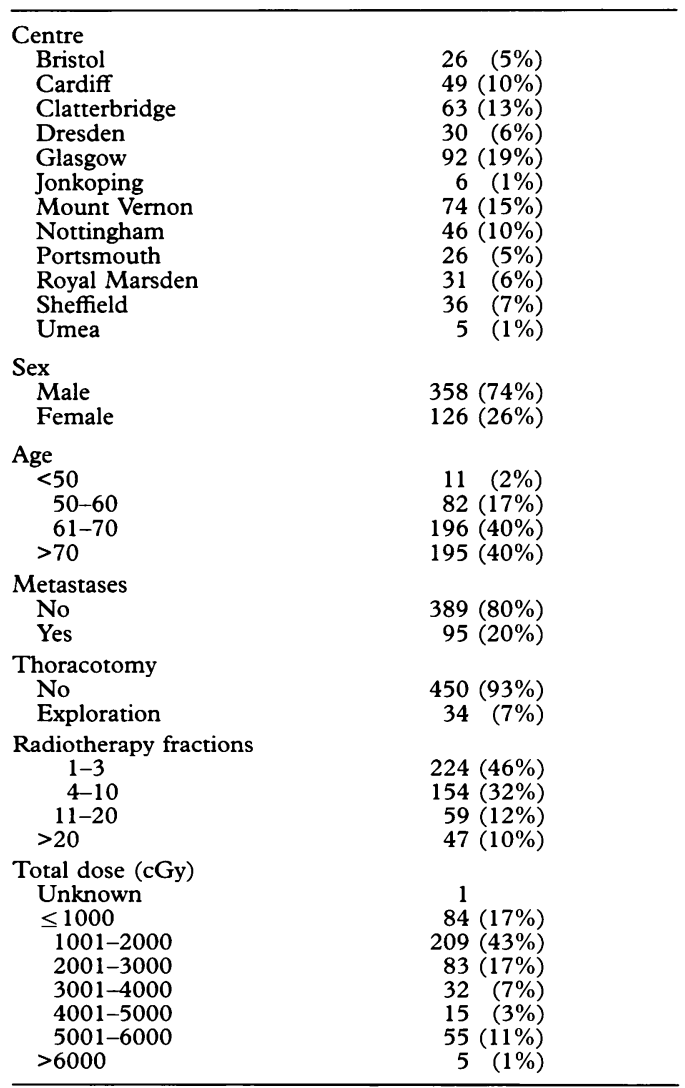




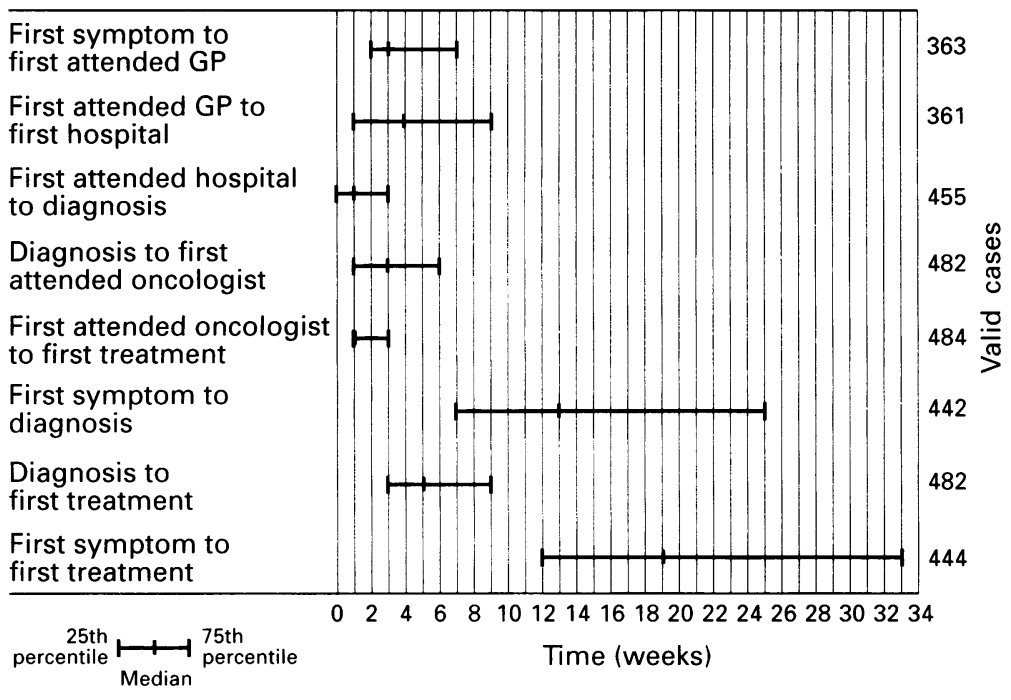

Figure 1 Time intervals from first symptom to attendance for treatment. Data shown as median and 25th and 75th percentiles.

\begin{tabular}{lr} 
Table 2 Inclusion in CHART trial \\
\hline Included in CHART trial \\
No & $460(95 \%)$ \\
Yes & $24(5 \%)$ \\
Principal reason for exclusion & \\
Poor general state & $170(37 \%)$ \\
Volume too large & $125(27 \%)$ \\
Metastases & $89(19 \%)$ \\
Consultant's decision & $14(3 \%)$ \\
Patient's decision & $12(3 \%)$ \\
Another/previous malignancy & $10(2 \%)$ \\
Previous radiotherapy/chemotherapy & $6(1 \%)$ \\
Inadequate histology & 6 \\
Pleural effusion & $5(1 \%)$ \\
Rib involvement & $2(<1 \%)$ \\
Poor pulmonary function & $2(<1 \%)$ \\
Other & $19(5 \%)$ \\
Unknown & 24 \\
\hline
\end{tabular}

to $46 \%$ of all patients; in $78 \%$ the fractionation was no greater than 10 .

Only 24 patients, $5 \%$ of all those surveyed, entered the CHART trial. The most frequent reasons for exclusion were poor general condition $(37 \%)$, too large a volume of the chest requiring treatment $(27 \%)$, and the presence of metastases outside the chest $(19 \%)$ (table 2).

The time intervals for all phases from onset of first symptom to first treatment by radiotherapy are shown in fig 1 . Because it was possible to gather only the month of presentation of first symptom we have made a calculation based in all cases upon the 15 th day of the month. A total of 418 patients had their initial consultation with a chest physician in hospital, of which 104 were referred to a chest surgeon. In all, 282 patients were referred to an oncologist by a chest physician, 143 by a chest surgeon, and 59 by other doctors including general practitioners.

\section{Discussion}

The median interval between first symptom and presentation to a general practitioner was three weeks. Many patients with lung cancer have pre-existing lung disease, often related to cigarette smoking, but it seems likely that an additional symptom such as haemoptysis, in- crease in severity of cough, shortness of breath, or superadded respiratory infection brought the patient to the family doctor. The median interval of four weeks from presentation to the general practitioner to first attendance at hospital appears satisfactory as it includes the delay between referral and clinic attendance. However, the time between onset of first symptom and first attendance at the general practitioner was not verified by referral to the general practitioners' notes.

The interval from first attendance at hospital to diagnosis again seems satisfactory but a greater interval appears to occur (median 3 weeks, range 1-6 weeks) between the time of the diagnosis and the patients' visit to a clinical oncologist. Perhaps in some cases this interval was affected by the commonly held view that nearly all patients with inoperable NSCLC should only receive radiotherapy for palliation of symptoms, and that a "watch" policy should be followed in those with no symptoms.

The median interval between first seeing an oncologist and attendance for first treatment was one week which seemed satisfactory, especially considering the heavy workload of many of the UK centres taking part in the CHART trial. As most of these patients were selected immediately for palliative rather than curative radiotherapy, the interval between first attendance and the start of treatment was not extended because of further investigation. The median interval between first attendance and the start of radical treatment was longer.

However, concentrating on median values diverts attention from the longer time intervals experienced by many patients at the various stages leading to treatment. A quarter of the patients experienced a delay of more than nine weeks between first attending their general practitioner and attending hospital. Also, more than a quarter of the patients did not see an oncologist until more than eight weeks after seeing their first hospital specialist.

The time observed for lung tumours to double their volume ranges from four to 56 weeks with a median of 17 weeks. ${ }^{89}$ With the faster growing tumours and the longer intervals in the process from initial referral to diagnosis and attendance for radiotherapy, a doubling of the bulk of the primary tumour may have occurred together with local and distant spread.

It is of interest that the Standing Medical Advisory Committee's Working Group in its report on the management of lung cancer recommends that, for patients where surgical resection is planned, no more than $6-8$ weeks should elapse between the patient's first presentation to the general practitioner and the operation. ${ }^{2}$ A similar recommendation ought to be appropriate for patients accepted for another type of radical treatment.

In general the number of attendances for radiotherapy in the UK centres was small. Nearly half the patients were treated in three or fewer fractions. This demonstrates the current practice for palliation in the UK centres which has been much influenced by the results of randomised controlled trials in palliative radiotherapy conducted by the Medical Research 
Council. These trials have shown that short term symptomatic control can be achieved equally well by giving radiotherapy in a few fractions over a short time interval as by giving long courses with many individual treatments. ${ }^{1011}$

The study excluded those cases where the primary tumour had been removed by surgery but included 34 patients whose tumours were found at operation to be inoperable. Of the 484 patients included $418(86 \%)$ were first seen at hospital by a chest physician; however, $143(30 \%)$ were referred to the oncologist by a chest surgeon. Only $59(12 \%)$ were referred to an oncologist by another doctor, usually the general practitioner. These data confirm the cardinal roles of the chest physician and chest surgeon in making management decisions for patients with lung cancer.

The interim report of the CHART randomised trials has shown significantly improved survival of the CHART treated cases compared with those treated by conventional radiotherapy ${ }^{7}$ with an improvement in the two year overall survival from $20 \%$ to $30 \%(p=0.006)$. This result encourages the use of radiotherapy and the CHART regime in advanced but apparently localised NSCLC in patients with good performance status. Of the large number of patients who presently receive palliative radiotherapy in the UK some might be suitable for more radical treatment if referred at an earlier stage in their illness. The detailed reasons for the long delays experienced by some of these patients between first symptom and start of treatment could usefully be the subject of prospective audits.

We are grateful to Jane Fallows for the preparation of fig 1 .

Oncology Centres contributing to the CHART Trial: Bristol Oncology Centre, Bristol; Beatson Oncology Centre, Glasgow Nottingham General Hospital, Nottingham; Royal Marsden Hospital, London and Surrey; Velindre Hospital, Cardiff; Clatterbridge Hospital, Merseyside; Cookridge Hospital, Leeds; St Mary's Hospital, Portsmouth; Weston Park Hospital, Sheffield; Mount Vernon Hospital Northwood; University Hospital, Umea; County Hospital, Ryhov-Jonkoping, and Carl Gustav, Dresden.

1 Cancer Research Campaign Factsheet 5.2. Cancer in the European Community: 1992.

2 Standing Medical Advisory Committee Report on Working Party chaired by Whitehouse JMA. Management of lung cancer: current clinical practices. 1994

3 Edinburgh Lung Group. Patients presenting with lung cancer in South East Scotland. Thorax 1987;42:843-7.

4 Cancer Research Campaign Factsheet 11.5. Lung cancer and smoking. 1992

5 Noordijk EM, Poest Clement EVD, Hermans J, Wever AMJ, Leer WJH. Radiotherapy as an alternative to surgery in elderly patients with resectable lung cancer. Radiother in elderly patients with

6 Saunders MI, Lyn BE, Dische S. Continuous, hyperfractionated, accelerated radiotherapy (CHART) in nonsmall cell lung cancer. Lung Cancer 1993;9:221-8.

7 Saunders MI, Dische S, Barrett A, Parmar MBK, Harvey A, Gibson D. Randomised multicentre trials of CHART versus conventional radiotherapy in head and neck and non-small cell lung cancer. An interim report. $\mathrm{Br} f \mathrm{Cancer}$ 1996;73:1455-62.

8 Weiss W. Peripheral measurable bronchogenic carcinoma. Am Rev Respir Dis 1971;103:198-208.

9 Steele JD, Buell P. Asymptomatic solitary pulmonary nodules - host survival, tumour site and growth rate. $\mathcal{F}$ Thorac Cardiovasc Surg 1971;65:140-51.

10 Medical Research Council. Inoperable non-small cell lung cancer. Medical Research Council randomised trial of palliative radiotherapy with two fractions or 10 fractions. palliative radiotherapy with tw
$B r \mathcal{F}$ Cancer 1991;63:265-70.

11 Medical Research Council. Medical Research Council randomised trial of palliative radiotherapy with two fractions or a single fraction in patients with inoperable non-smal cell lung cancer and poor performance status. $\mathrm{Br} f \mathrm{Cancer}$ 1992;65:934-41. 